\title{
LOISE DE ROSA, CUSTODE ASSAGII DELLA ZECCA DI NAPOLI, DAL TEMPO DI LADISLAO DI DURAZZO A QUELLO DI DON FERRANTE
}

LOISE DE ROSA, CUSTODIAN ASSAGII OF THE MINT OF NAPLES, SINCE LADISLAS OF DURACE TIME TO DON FERRANTE

Simonluca Perfetto

Società Numismatica Italiana Universidad Complutense de Madrid pesperfe@gmail.com

Resumen: A través de un privilegio preservado en el Archivo de la Corona de Aragón ha sido posible no solo reconstruir algunos eventos de la vida de Loise De Rosa, autor de los Ricordi, sino también descubrir la existencia del oficio de guardián de los assaggi de oro, de plata y cobre hechos en ceca de Nápoles. La documentación también permite analizar las características institucionales de este cargo que De Rosa ejerció durante el reinado de tres reyes angevinos y dos aragoneses.

Palabras clave: Loise De Rosa, ceca de Nápoles, guardián de los assaggi, Alfonso El Magnánimo, Corona de Aragón, Ricordi, siglo XV.
Abstract: Through a privilege preserved in the Archive of the Crown of Aragon it was possible not only to reconstruct some events of the life of Loise De Rosa, author of the Ricordi, but also to discover the existence of the office of keeper of the golden assaggi, silver and copper made in the mint of Naples. Furthermore the institutional features of this office are reconstructed, also because De Rosa exercised it during the reign of three Angevin rulers and two Aragoneses.

Keywords: Loise De Rosa, mint of Naples; keeper of the assaggi, Alfonso the Magnanimous, Crown of Aragon, Ricordi, $15^{\text {th }}$ century. 
Qualunque zecca medievale europea doveva inevitabilmente servirsi di un mastro di prova o di un ufficiale deputato a saggiare la bontà dei metalli ( $m a-$ gister probe o assayador), per far sì che la moneta del caso fosse coniata secondo la lega prescritta dal Papa, dal re, dalla città, dal barone e così via. ${ }^{1}$

L'importanza di questa fase produttiva della moneta era tale che in caso di mancanza di un mastro di prova, nella migliore delle ipotesi si sarebbe avuta una zecca di moneta falsa o falsata, mentre in caso di assenza di un qualsiasi altro ufficiale, mastro di zecca incluso, vale a dire l'appaltatore della zecca, questa avrebbe potuto funzionare ugualmente con risultati corretti. Per tali ragioni, gli esiti dei lavori della prova, cioè i saggi, meritarono l'istituzione di un custode del cosiddetto assagium. ${ }^{2}$

Questo ruolo, che da un punto di vista documentale sembra configurare apparentemente l'antesignano dell'ufficio di guarda prova, che in epoca moderna in particolare dal 1503 risultava sempre presente a Napoli, ${ }^{3}$ non può considerarsi propriamente tale, ma prima di esaminarlo unitamente alla figura di un suo celebre esercente, bisogna commentare l'interessante struttura del privilegio aragonese che aveva rinnovato in tale carica l'ufficiale de quo.

Il documento fa parte della cancelleria napoletana di Alfonso il Magnanimo i cui registri, su richiesta di Giovanni II, tra il 1458 e il 1467 furono tras-

1 Il mastro di prova era il cosiddetto 'ensayador', vale a dire «el técnico capacitado para conocer la composición de los metales» (tratto da Torres Lázaro, 1998, I: 19).

Abbreviazioni: ACA = Barcelona, Archivo de la Corona de Aragón; AGS = Valladolid, Archivo General de Simancas; AGV = València, Archivo General del Regne de València $;$ ASNA = Napoli, Archivio di Stato di Napoli; RCS = Regia Camera della Sommaria.

2 Non è ben chiaro il momento dell'istituzione di questo ufficiale a livello europeo e tantomeno a Napoli, ma questa figura pare piuttosto antica, giacché un guardiano dei saggi risultava esistente in Inghilterra già intorno al 1282-1283, sotto Edoardo I (Lawson, 1857: 670), datazione che induce a credere presenze di uffici simili e coevi in gran parte d'Europa. Secondo le regole in uso nel mondo catalano (segnatamente a València), almeno dal tempo di Pietro il Cerimonioso nel 1369, esisteva un guarda (Mateu y Llopis, 1937: 98 e 107), «empleat de la seca encarregat de fer l'aliatge i custodiar tota la fabricació de la moneda». In realtà furono almeno «dos homens bons e aptes per a guardes» (Mateu y Llopis, 1937: 112) di tutte le operazioni legate a ogni ufficio della zecca di València.

3 Da Simancas, A(rchivo) G(eneral) de S(imancas), Visitas de Italia, leg. 78, exp. 22, anno 1584, Hec deducit Joseph de Ancora in eius defensionibus circa caput ei datum in Regia Visita, senza numerazione, cap. 1: «l'officio di esso Gioseppo chiamato Guarda prova consiste in assistere, et intervenire quando la moneta dopoi lavorata, che si vuol liberare, et uscire da detta regia Zeccha se ne fa prova, et all'hora intervene, et assiste esso Gioseppo come guarda prova mentre si fa la detta prova per il mastro di prova e dopoi fatta, et giudicata per bona come la Regia Corte ha comandato, esso Gioseppo ne fa fede, dentro la quale si conserva la detta prova, e dopoi la pone dentro una cascia chiamata la guarda prova con sei chiave, delle quali tre ne tene esso Gioseppo e tre altre il mastro di prova; nel quale esercitio assiste, e bisogna assistere esso Gioseppo per obligo del suo officio; et non in altro, il che è vero etc.» Per un elenco degli ufficiali della zecca si veda Perfetto, 2020a: 7-40. 
messi a Barcellona, ove oggi sono conservati. ${ }^{4}$ Il 7 febbraio 1443, il re si trovava a Benevento, dove appunto aveva segnato, tra gli altri, il privilegio che rinnovava a Loise De Rosa lo "officium custodis assagii seu probe monete auree, argentee et heree cudende in Sicla civitatis nostre Neapolis». ${ }^{5}$ Questo noto personaggio (vd infra) aveva ricoperto il detto ufficio dai tempi di Ladislao, per cui in una fase temporale più o meno ampia, ma comunque collocata ante 1414, ${ }^{6}$ nonché durante il regno di Giovanna II (1414-1435). Nel privilegio, non si fa cenno, invece, al regno di Renato d'Angiò (1435-1442), durante il quale l'ufficio fu certamente esercitato.

Questa impostazione mostra il sottile filo diplomatico che la corte alfonsina percorse nei primi anni di insediamento a Napoli, giacché è evidente che i richiami storici stavano a sottolineare l'ereditarietà del Regno di Sicilia citra Pharum, che era passata attraverso l'istituzione di Alfonso, quale erede, da parte di Giovanna II al tempo del vicariato (1421-1423). Non solo, v'è di più: è possibile che Loise De Rosa, confermato nell'ufficio l'11 febbraio 1420 da Giovanna II e riconfermato 1'8 agosto 1422 da Gaeta, abbia ricevuto quest'ultima concessione proprio da Alfonso in qualità di vicario. ${ }^{7}$ Ciò denoterebbe che le attività svolte durante il vicariato non caddero totalmente nell'oblio, ma furono recuperate per quanto possibile, dopo la conquista. ${ }^{8}$

4 ACA, Cancillería, Registros 2902, Privilegiorum Cancilleriae Neapoli I, Pro Loyse de Rosa, anni 1440-1444 (Benevento, 7 febbraio 1443); f. 159v. Il privilegio è interamente trascritto in appendice. Oggi esistono i regesti della cancelleria di Alfonso il Magnanimo recentemente pubblicati in López Rodríguez e Palmieri (2018), ove detto privilegio è regestato a p. 40, con n. 170. Prima che l'inventario fosse pubblicato, avevo pazientemente selezionato i pochi privilegi riguardanti zecche e monete, avviandone le rispettive edizioni critiche, delle quali per ora è venuta alla luce quella su Benedetto Cotrugli (Perfetto, 2016: 14-16), che nel nuovo inventario si trova regestato a p. 560 con n. 83. Palmieri (2018: VIII) ricorda che non vi fu alcuna radicale distruzione dei registri di Alfonso I conservati a Napoli, durante l'ultimo grande conflitto, per cui i volumi custoditi in ACA sono gli originali, ivi inviati per il fratello del Magnanimo, Giovanni II. Il Moscati (1953) aveva intrapreso per primo la ricerca di questi registri a Barcellona.

5 Al contrario di quanto noto al mondo numismatico fino al 2018, le mansioni dell'ufficio evidenziano altresì che al tempo di Ladislao di Durazzo e di Giovanna II si coniarono a Napoli monete d'oro e d'argento.

6 Loise De Rosa nacque a Pozzuoli nel 1385, per cui potenzialmente poté ricoprire questo ufficio sin dai primi anni del Quattrocento, come era costume all'epoca (si pensi all'archivario Annibale Mastrogiudice, che ottenne la guida dell'Archivio della Regia Zecca a sedici anni circa, cfr. Perfetto, 2019a: 254)

7 I provvedimenti presi in qualità di vicario conservavano ancora la loro natura angioina e la prima intestazione degli stessi era sempre a nome di Giovanna II, benché li sottoscrivesse il Magnanimo.

8 Tale schema storico-diplomatico, ricorrente in molti casi, è lo stesso che autorizza a credere nell'operatività della zecca di Lanciano nel triennio 1421-1423, riconfermata in ordine cronologico come prima zecca del Regno abilitata a battere oro, nel 1441, stante la provvisorietà e la successiva dismissione di quella di Gaeta in favore di Napoli (sullo spostamento: Silvestri, 1959: 603-604). 
Il mancato riferimento a Renato d'Angiò reca invece un ampio ventaglio di spiegazioni. A tal proposito, si ricorda che le modalità operative di tipo politico-economico adottate durante il regno di Giovanna II furono traghettate come immutate insino alla venuta di Renato, ${ }^{9}$ per volere della regina, che per conseguire questo scopo aveva incaricato Gaspare Bonciani, il primo ministro del Regno..$^{10}$ Recentemente, grazie a una importante lettera, abbinata a un denaro di Renato d'Angiò, è stato possibile dedurre che quest'ultimo era stato scelto quale erede definitivo di Giovanna II, già nel 1432, e che quindi Luigi III d'Angiò non fu sostituito da Renato a seguito di morte sopravvenuta nel 1434 , come la storiografia ha tramandato. ${ }^{11}$

Di conseguenza, il primo motivo che Alfonso ebbe nel non richiamare la memoria di Renato nel privilegio fu la questione ereditaria, sebbene al momento della conquista, avesse ritrovato una Napoli organizzata come quella dei tempi di Giovanna II, allorquando fu vicario. Sulla base di ciò, Loise De Rosa, negli anni di Renato, avrebbe dovuto ricoprire de plano il detto ufficio nel segno della continuità, ma il riferimento, che il privilegio fa alla iusta sincera devocione et fide grata quoque grandia utilia fructuosa et accepta servicia, lascia ipotizzare anche la possibilità che costui si fosse schierato a favore di Alfonso già a partire dal 1436 e che, per tale motivo, non abbia effettivamente ricoperto l'ufficio durante l'ultimo regno angioino. Del resto, i principali beneficiari delle concessioni alfonsine seguite al parlamento del 1443 annoveravano i fautori filo-aragonesi della conquista di Napoli. Ciò detto, la soluzione preferibile rimane quella della voluta omissione diplomatica del nome di Renato, in quanto lo stesso Loise De Rosa, nella sua unica opera di Ricordi, per gloria personale, racconta d'aver servito sei regine e sette re, tra i quali Luigi III e Renato d'Angiò, ${ }^{12}$ nonché di essersi addirittura ribellato ad Alfonso al tempo del vicariato, notizia sfuggita agli studiosi del personaggio:

9 Iadanza, 1952: 20.

10 Perfetto, 2019: 238-239.

11 Perfetto, 2019: 260-262 e nt 168.

12 Petrocchi, 1957: 535. Riporto il testo dalla trascrizione di Formentin (1998, II: 513): «A(n)no D(omi) ne $\mathrm{m} \mathrm{c}^{\mathrm{iiij}}$ lij yo, Loyse De Rosa, aio (com)menczato chisto libro et só omo / de a(n)ne lxvij; et in chisto tienpo aio serbute dudice riale, sey ry et sey / rigine. Ly ry do chiste: re Odo quando ve(n)ne a la incoronacione de re / Lanczala' a Gageta; lo secundo re Lanczalao; lo tierczo re Iacobo; lo // quarto re Loyse; lo quinto re Renato; lo siesto re Alfonczo. Et / sonce le infornate che no (n)ce so (con)tate: aio serbuto re Fe(r)rante / et anco lo gra' senesscarco che sengnioriava tutto chisto Riame ma / no era re». I re indicati dunque erano: Ottone di Brunswick, ultimo marito di Giovanna I, Ladislao, Giacomo II di Borbone, marito di Giovanna II, Luigi III d'Angiò, Renato d'Angiò, Alfonso e Ferdinando d'Aragona. Su Ottone v. nt 19. 
Po' ve(n)ne lo duca d'Enyoya et arcune singniure se rebellaro (con)tra la re//gina. ${ }^{13}$ Et in chillo tienpo ve(n)ne re Alfonso et cacczao lo duca d'Enyoya $^{14}$ / et multy singniure se rebbelaro a la regina et derose ${ }^{15}$ allo re Alfonso. / Et depo' che la regina Ioa(n)na murio et lo re restao unico singniore, v/olce et (com)mandao a ttutty ly singniure che iorassero umaio ${ }^{16} \mathrm{a}$ don Ferrante: et cussy fo fatto.

Quando nel 1421 molti Signori si ribellarono a Giovanna II in favore di Alfonso, che aveva cacciato Luigi III d'Angiò, il «derose» (= De Rosa) mantenne fede alla stessa, ribellandosi proprio ad Alfonso. Questo riferimento storico conferma sia la proverbiale magnanimità del nuovo sovrano catalanoaragonese, sia l'ostinata continuità ereditaria con la quale evidentemente il re pretese di novare per gran parte la precedente corte di cui riteneva propri i relativi diritti (quod est firmum confirmatur), sia il voluto oblio diplomatico di re Renato.

Grazie ai suoi Ricordi, cronaca scritta in tarda età, tra il 1452 e il $1475,{ }^{17}$ Loise De Rosa è diventato un personaggio piuttosto famoso, nonostante la sua singolare opera letteraria sia stata sottovalutata a cavallo tra Ottocento e Novecento. ${ }^{18}$ In particolare, al singolare autore è stata contestata l'eccessiva autostima e la fantasia usate nell'attribuirsi alti ruoli per i quali sussiste dubbio sul fatto che egli abbia potuto ricoprirli, perché in realtà fu sostanzialmente il mastro di casa di molti dignitari del Regno, nonché cerimoniere in occasioni importanti. Questo incarico aveva avuto origine in giovanissima

13 Deve dedursi che la regina fosse Giovanna II d'Angiò-Durazzo e non Isabella di Lorena, moglie di Renato, considerato che, poco più avanti, il De Rosa cita l'arrivo di Alfonso nell'atto di scacciare Luigi III dal Regno, avvenimenti che precedono la morte della regina Giovanna riferita qualche battuta più tardi. D'altra parte alla carta 47r, quella successiva, il De Rosa definisce Renato come re, per cui non possono sussistere equivoci tra Luigi, Renato e Giovanni d'Angiò che furono tutti duchi «d'Enyoya».

14 Il riferimento è diretto alla desistenza di Luigi III d'Angiò nel luglio del 1421, quando Alfonso entrò a Napoli.

15 Questo passo dei Ricordi è tratto da Formentin (1998, II: 629, f. 46v.), ove però il termine 'derose' non si rinviene né nel glossario (II: 758-759, 796, 844), né nell'indice (II: 891, 897-898) e nemmeno nella biografia del De Rosa (I: 19-23). Probabilmente non è stato valutato come il nome dell'autore.

16 Si tratta del ligio omaggio che università e baroni prestavano al sovrano. La cerimonia comprendeva il solenne giuramento di fedeltà al sovrano aragonese.

17 Il 1452 può considerarsi il primo anno di stesura; successivamente l'autore ne scrisse il seguito e, nell'ultima parte della vita, operò integrazioni senza particolare cura nell'evitare contraddizioni o nel tornire l'armonizzazione dei novi inserimenti (Formentin, 1998, I: 48-49).

18 Ne parlò anche il Croce, 1913: 260-263, nonché passim. Secondo Bentley (1995: 22), quella di Croce sarebbe la migliore discussione sul De Rosa, ma anche dal giudizio espresso in Formentin (1998, I: 16-17), la stessa sarebbe quella più affabile. 
età, a soli cinque anni, probabilmente nelle forme che si confacevano a un bambino, quando fu mandato a servire Ottone IV di Brunswick-Grubenhagen che, nel 1390, aveva partecipato all'incoronazione di Ladislao di Durazzo a Gaeta ${ }^{19}$.

Tuttavia, il giudizio sul suo conto è maturato unicamente in base alla sua opera letteraria, della quale vengono messi in dubbio gli incarichi indicati e grazie alla quale si constata la bassa cultura dell'autore, facendogli quasi una colpa per aver riportato i fatti antecedenti a Carlo III di Durazzo, solo in base alla tradizione orale paterna, e per esser stato testimone diretto dei fatti narrati per il periodo successivo. ${ }^{20}$

A contrario, alla luce del documento presentato in questa sede, quand'anche non si possano negare sia la bassa cultura di Loise De Rosa, status che però sarebbe meglio definire come 'peculiare', rappresentando questo un coacervo culturale di natura popolare e cortigiana, di ispirazione angioina e aragonese, di improprie scelte linguistiche, adottate però secondo un preciso elemento psicologico-popolare, sia alcune amnesie storiche ${ }^{21}$, questo personaggio può essere rivalutato unitamente alla sua opera. Infatti, il privilegio conferma i grandissimi servizi svolti dal De Rosa per tre sovrani almeno, rispettivamente Ladislao, Giovanna II e Alfonso il Magnanimo; e lo fa con tenore altisonante, ma soprattutto promanante dalla Corte e non dall'interessato, restituendo in tal guisa un quadro che pertanto accredita le vanterie riferite nella sua opera. ${ }^{22}$ In secondo luogo, tra i numerosi incarichi che egli enumera nei Ricordi, sorprendentemente omette di citare proprio l'ufficio di custode degli assaggi, particolare che fa emergere che semmai avesse esagerato sul proprio conto, lo avrebbe fatto non già in eccesso, bensì per difetto.

19 «Ly ry so chiste: re Odo quando ve(n)ne a la incoronacione de re / Lanczala' a Gageta», trascritto in Formentin (1998, I: 513). Anche se Ottone non era più re, l'autore lo considera tale. Quasi gli riconosce un titolo da re emerito.

20 Cfr. Croce, 1913: p. 264.

21 Ad esempio, chiama Federico II col nome del nonno, Federico Barbarossa, errore peraltro comune all'epoca anche tra gli storici, ma ne colloca correttamente i fatti del 1229.

22 Si dichiara mastro di casa di re Giacomo II, del gran siniscalco (individuato dagli storici come Sergianni Caracciolo, ma potrebbe trattarsi di Innico, presente sul letto di morte del re, nel 1458, o di Pietro de Guevara, visto l'abbinamento a re Ferrante fatto dal De Rosa) dei cardinali Latino Orsini e Ugo di Lusignano di Cipro, del patriarca d'Alessandria Giovanni Vitelleschi, dei principi di Salerno, degli Orsini e dei Sanseverino, dei duchi di Sora e del Vasto (Guevara appunto), dei conti di Troia e di Ariano, di Cola d'Alagno e di altri signori. Fu viceré del contado di Bisceglie e di Val di Gaudo, due volte viceammiraglio, la prima per conto di Giovanni Fregoso, fratello del doge di Genova, la seconda per conto del principe di Salerno (De Nichilo, 1991). 
Probabilmente, aveva ricoperto talmente tanti incarichi che a ottant'anni suonati, mentre scriveva, non poté ricordarli al completo, benché quello di custode degli assaggi assurgesse ad un ufficio con propri gagi, lucri e debiti, che esulavano da quelli percepiti per essere mastro di casa. Quest'ultimo incarico, però, assorbiva di fatto la maggior parte del tempo al De Rosa, per cui va precisato che quella di custode non poteva rappresentare la sua principale attività nella Curia regia, in quanto detto incarico non prevedeva la stabile presenza in zecca, ma semplicemente il ritiro dei saggi a orari stabiliti e per di più con la possibilità di essere sostituito (v. infra).

Un primo tentativo di ricostruzione delle cariche del De Rosa è stato avviato dal De Blasiis, il quale però ha dubitato del fatto che gli omonimi che aveva individuato potessero coincidere col nostro anziano narratore. ${ }^{23}$ Tuttavia, almeno per quanto concerne la notizia di un ufficio della Regia Zecca di Napoli concesso a Antonello Fondacaro, ma olim appartenuto a Loise De Rosa, è possibile confermare inequivocabilmente l'identità col nostro personaggio. ${ }^{24}$ Infatti, il Fondacaro risulta maestro di prova della zecca di Napoli tra il 1467 e il $1468,{ }^{25}$ per cui dové tenere l'ufficio del De Rosa tra il 1459 e il 1467 , essendo incompatibile l'esercizio di entrambi.

L'ufficio di custode era stato concesso al De Rosa ad vitam, per cui è da ritenere che lo esercitò anche al tempo di Ferrante, quanto meno agli inizi del suo regno, poiché l'incarico non necessitava di un rinnovo a ogni successione dinastica. ${ }^{26}$ Tuttavia, ricevutolo con la potestas substituendi, è possibile che di fatto l'ufficio venisse esercitato da un sostituto, specialmente nei momenti in cui il De Rosa fu maggiormente impegnato nelle cerimonie, nonché nella parte della propria vita, in cui cominciò a scrivere i Ricordi (1452-1460), allorquando si può immaginare un maggiore impegno presso $\mathrm{i}$ propri padroni e un coinvolgimento diretto nel tramandare taluni fatti stori-

23 Per le apparizioni documentali del De Rosa, si rimanda a De Blasiis (1879: 412-414), nonché a Formentin (1998, I: 13-14) che vi si riporta e lo aggiorna.

24 La notizia della quale il De Blasiis (1879: 413), ha riportato il regesto è oggi perduta, ma si trovava in ASNA, RCS, Executoriarum, anni 1442-1460.

25 Il documento riguardante il Fondacaro, in qualità di mastro di prova, è integralmente trascritto in BOVI 1968, p. 65 e si trova in ASNA, RCS, Significatoriarum, I (Napoli, 3 agosto 1467), ff. 195v-196r; richiamato anche in Perfetto (2012: 47, nt 22).

26 Infatti, gli anni dell'esecutoria in favore del Fondacaro (1442-1460) lasciano aperta tale possibilità tra il 1458 e il 1460. Invero, due possono essere le soluzioni al caso: poco dopo l'insediamento di Ferrante al trono di Napoli, l'ufficio fu probabilmente alienato direttamente dal De Rosa, ovvero fu richiesto dalla Corte per essere ex novo concesso. 
ci. Si ricorda infine che, in caso di sostituzione, i lucri derivanti dall'ufficio venivano generalmente divisi a metà tra proprietario e sostituto, salvo diversa convenzione, ${ }^{27}$ mentre tale pratica fu assolutamente vietata per l'ufficio di maestro di prova, che richiedeva una particolare abilità esecutiva nonché una certa integrità morale..$^{28}$

Attraverso la disamina di importanti fonti correlate alla Zecca della Moneta di Napoli, si è potuto rilevare il totale distacco del De Rosa da questa istituzione, per lo meno da un punto di vista documentale, nonostante ricoprisse il predetto ufficio di custode. ${ }^{29}$ Inoltre, egli non ebbe frequenti rapporti con gli avventori della zecca, ${ }^{30}$ pratica al contrario comune per l'epoca tra gli ufficiali e i mercatanti, ${ }^{31}$ né pare aver avuto rapporti con banchieri, ${ }^{32}$ salvo la nota di un conto che si trova proprio nei Ricordi e che si riferisce tra gli altri a Salvatore de Ponte, che fu maestro della zecca di Napoli almeno nel $1461 .^{33}$

Infatti, scrive il Formentin: «Alla mano principale [vale a dire quella del De Rosa] si deve l'annotazione che si legge a c. 75v., l'ultima carta di guardia del manoscritto: è una lista di quattro nomi - Andrea Brussca, Andrea De Ponte, Piero De Ponte, Sarvatore De Pente (sic una macchia copre la -e di Sarvatore) - , accanto ad ognuno dei quali è registrata una somma (rispettivamente due once e quindici tarì, un'oncia e venti tarì, un'oncia, venticinque tarì); sotto si dà l'ammontare complessivo (sei once). Anche in questo caso, probabilmente, come per la serie alfabetica di c. 66r., la nota era già stata scritta quando il foglio venne riutilizzato come carta di guardia $»^{34}$. Dunque il conto appare così schematizzato:

27 Oppure venivano stipulate delle convenzioni attraverso le quali gli emolumenti dell'ufficio venivano ripartiti secondo l'accordo tra il titolare e il sostituto. Molti casi per la Regia Zecca di Napoli, di qualche decennio posteriori, sono documentati nel fondo AGS, Visitas de Italia, leg. 16.

28 Sulla potestas substituendi nell'ufficio di mastro di prova, $c f r$. Perfetto, 2012: 49-55.

29 Il De Rosa non è mai menzionato nella cospicua mole di documenti sulla zecca di Napoli e nemmeno tra i testimoni dei documenti segnati a Napoli per le zecche catalane, che Crusafont i Sabater (2015: 557-576 e 683-691) ha pazientemente raccolto.

30 Non è annoverato nel Llibre de Comptes de la seca de Nàpols studiato e trascritto in Perfetto (2015: 172-243). Manca anche nella recente raccolta di nomi di ufficiali della zecca di Napoli, contenuta in Vall-1losera i Tarrés, (2016: 78-100).

31 La pratica era comune al punto tale da aver creato abusi sull'acquisto e la vendita dei metalli da introdurre in zecca, per cui al tempo nel 1561 fu vietata agli ufficiali: «non facciano mercatura d'oro né argento» (Perfetto, 2017: 130).

32 Non è annoverato nel Compte del Banc d.en Mirabal studiato e trascritto in Navarro Espinach e Igual Luis (2002: 71-205).

33 Formentin, 1998, II: 697, f. 75v.; Perfetto, 2016a: 146-147, nt 8.

34 Tratto da Formentin (1998, II: 697). 


\begin{tabular}{|ll|}
\hline Andrea Brussca, & due once e quindici tarì \\
\hline Andrea De Ponte & un'oncia e venti tarì \\
\hline Piero De Ponte & un'oncia \\
\hline Sarvatore De Pente & venticinque tarì \\
\hline & 6 once \\
\hline
\end{tabular}

L'importo di 6 once non può rappresentare il totale che ammonterebbe a circa $543 \mathrm{~g}$, vale a dire a più di venti once ${ }^{35}$ ma può rappresentare un ulteriore importo riferito all'ultimo della lista, Salvatore de Ponte appunto, ovvero al De Rosa stesso che, prendendo nota per sé, non si è firmato in corrispondenza dell'importo. Di cosa si trattava? Innanzitutto si trattava di argento, in considerazione della presenza dei tarì che, benché di conto, non possono riferirsi ai tarì d'oro dei tempi di Federico II, i quali tuttavia erano ancora indicati nella comminazione delle pene degli statut ${ }^{36}$. Inoltre, considerati i personaggi e l'incarico del De Rosa in zecca, è molto probabile che si sia trattato di un'operazione collegata a questa istituzione ${ }^{37}$ In chiave contabile, dette annotazioni non potevano costituire operazioni di 'dare' provenienti dalla zecca, perché queste sarebbero state annotate in moneta effettiva, che il mastro di zecca o il credenziere avrebbero riconsegnato ai proprietari del metallo con un piccolo disavanzo, espresso in grana. Ad esempio, ciò accadeva quando il disavanzo era inferiore ai $3,60 \mathrm{~g}$, peso minimo per coniare un carlino. ${ }^{38} \mathrm{Per}$ converso, trattandosi di argento in once e argento in moneta di conto, v'è assoluta compatibilità con operazioni di zecca in 'avere'. Di conseguenza il De Rosa si era prestato come maestro di banca, vale a dire come colui che in zecca riceve i metalli dai mercanti. Quindi egli stesso, ricevuti dai mercanti indicati i metalli al di fuori della zecca, li aveva conferiti in loco, dovendolo praticare per le ragioni del proprio ufficio. Pertanto tali annotazioni, benché successive alla prima stesura del manoscritto (1452), ${ }^{39}$ possono considerarsi

35 Un'oncia equivaleva a 30 trappesi e pesava circa $0,0267 \mathrm{~kg}$.

36 Sul punto, Travaini, 2007: 95.

37 L'anno 1461, relativo all'attività in zecca del De Ponte, spinge a credere che il De Rosa avesse esercitato ancora il proprio ufficio fino al termine del 1460, in compatibilità con la datazione del registro Executoriarum citato supra, e che si fosse fatto coniare 6 once di tarì, una moneta oggi molto rara e dal carattere altamente celebrativo.

38 Un carlino equivaleva a 10 grani.

39 Formentin, 1998, II: 697. 
precedenti all'incarico di Salvatore de Ponte (1461), che in quella fase, a livello familiare, cominciò ad avere relazioni con la moneta da coniare.

Infine, un ulteriore indizio che collega l'autore dei Ricordi alla zecca è fornito da alcuni fogli di quest'opera, relativi alla parte in cui il narratore racconta non solo della coniazione di moneta a Napoli ad opera di Federico II, ma anche della tipologia del tornese, che si coniò a partire dall'epoca sveva e che si continuava a coniare ancora ai suoi tempi. ${ }^{40}$ Ciò dimostra che il menante conosceva bene l'ambiente legato alla zecca, per esserne appunto un ufficiale.

Ad ogni modo, il privilegio elargito al De Rosa non indica le precise mansioni che l'esercente dell'ufficio doveva svolgere. Tuttavia, attraverso le Instrucciónes para la cecca de la moneda, predisposte dal 1543 al 1561 sulla base dei principi catalano-aragonesi dei tempi di Alfonso, nonché attraverso i processi delle Visitas de Italia contro i guarda prove e a mezzo di qualche fonte relativa a zecche diverse da quella di Napoli, si possono ricavare alcune notizie. $^{41}$

L'innovazione apportata dalle Instrucciónes all'ufficio risiede probabilmente nell'assistenza che il guarda prove doveva prestare durante lo svolgimento della prova. Fatto ciò, avrebbe dovuto conservare gli assaggi dentro una cascia chiamata la guarda prova con sei chiave. Probabilmente al De Rosa, nel secolo precedente, fu richiesto solo quest'ultimo compito. Infatti, la riforma di epoca toletana era intervenuta per reprimere taluni abusi che potevano verificarsi già al momento del saggio del metallo e, per questo, non era più sufficiente il semplice ritiro dei saggi per la mera custodia. Pertanto l'ufficio di custode sarebbe stato assorbito da quello di guarda prove, ma in realtà non è così. Infatti il capitolo nono delle istruzioni recita:

Che la cassa del guarda prova stia et se debbia tenere in la cassa della Regia Zecca et se consigni al conservatore di esse prove il quale habbia da tenere una chiave di essa et l'altra lo predetto mastro de prova. ${ }^{42}$

40 Cfr. Formentin (1998, II: 599-600) e Perfetto (2020: 35-40).

41 Trascrizione e edizione critica delle Instrucciónes si trovano in Perfetto (2017). Utilissimi come confronti esterni sono l'Ordenacio de les coses que son necessaries en tota seca e il De ffondicio per fer la moneda menuda o d'argent, conservati in AGV, Titulos y Enajenaciones, vol. II, anno 1417, ff. 228 230 e trascritti da Mateu i Llopis (1937: 112-117).

42 Tratto da Perfetto (2017: 114), AGS, Visitas de Italia, leg. 16, exp. 17, Instrucciónes del 13 giugno 1543 , cap. 9 , f. 2 r 
Quindi l'ufficio del De Rosa, assimilabile a quello di «conservatore di esse prove», fu mantenuto in essere anche nel Cinquecento, mentre l'ufficio di guarda prova fu introdotto in posizione interposta tra il tempo che intercorreva per la realizzazione dei saggi e la definitiva consegna degli stessi al custode/ conservatore, al fine di monitorare tutte le fasi.

Tuttavia, al capitolo primo delle medesime istruzioni del 1543, allo stesso conservatore delle prove veniva ordinato di intervenire alla prova:

1. In primis volemo et cossì ordinamo et comandamo che allo offitiale nominato mastro de prova sia dato loco comodo in una casa de quessa Regia Zecca dove senza inpedimento possa fare detta prova et che tanto in lo fare de detta prova quanto della liberatione delle monete cossì de oro come de argento habbia da intervenire il regio conservatore delle prove et che li particulari che portarando oro, et argento in essa Regia Zecca possano intervenire alle prove de oro et de argento portarando per loro interesse. Verum se debbia stare allo che iodicarà esso mastro de prova, deputato per la Regia Corte. $^{43}$

In pratica le mansioni del conservatore erano le medesime del guarda prova, per cui bisogna concluderne che l'ufficio di guarda prova costituiva una rielaborazione più evoluta dell'ufficio di custode tenuto dal De Rosa, ma non sempre i due ruoli furono riuniti nello stesso soggetto.

La circoscrizione del ruolo che realmente fu affidato al De Rosa potrebbe meglio definirsi, attraverso l'accostamento a una sola fase dei numerosi lavori di controllo operati dalle guardes della zecca di València:

xij. E los dits monederos deven monedar la dita moneda. E apres de la dita moneda amonedada deven los dits moneders lljurar a les guardes una moneda o dos per a ffer lo assay de aquella e les guardes deven aquella moneda metre en un paper e deven lo estogar en una caxa e deu esser escrit assay de tal delliurança segons sera dit davall pus llarch. ${ }^{44}$

Il raccordo tecnico-produttivo della moneta tra i 'guardiani catalani' e i 'custodi degli assaggi napoletani' di evidente matrice angioina, si ebbe solo

43 Tratto da $i v i$ (p. 112), cap. 1, f. 1 r.

44 Da AGV, Títulos y Enajenaciones, vol. II, anno 1417, De ffondicio per fer la moneda menuda o d'argent, cap. 12, secondo il transunto di Mateu y Llopis (1937: 114). Per questi custodi, la protezione dei saggi e dei campioni in una cassa (l'officio del De Rosa) rappresenta un compito marginale, poiché essi assumono a tratti le funzioni dei più evoluti 'comprobatori' e 'giudici delle differenze', che a Napoli saranno istituiti solo nel XVI secolo. 
con la riforma di Don Pietro di Toledo, che istituì guarda prova, comprobatore e giudice delle differenze, peraltro, a mio avviso, all'esito di un profondo studio degli istituti adottati dal Magnanimo nel Mediterraneo aragonese.

Quanto agli strumenti di cui il De Rosa doveva essere dotato per esercitare correttamente l'ufficio, si può dedurre che avesse bisogno di una cassetta munita di chiavi. Almeno una chiave per sé medesimo e una per il mastro di prova. Nel 1458, una cassetta con «certi assaggi di moneta d'argento e di oro coniata in Aquila» fu inviata presso la Regia Camera della Sommaria, affinché il suo contenuto fosse verificato dall'allora maestro di prova, Giliforte d'Urso. ${ }^{45}$ È possibile che questa cassetta, benché di provenienza aquilana, sia stata presa in carico proprio da Loise De Rosa per essere trasportata dalla Sommaria, ove era pervenuta, al maestro di prova presso la zecca, ufficiale dal quale il custode dipendeva.

In conclusione, le innumerevoli cariche ostentate dal De Rosa, nei suoi Ricordi, non meritano di essere screditate, bensì di essere ricostruite ed approfondite. Queste infatti consentirebbero di conseguire significativi apporti per lo studio delle Corti angioine e aragonesi, della loro successione nel tempo, nonché delle fusioni dell'apparato burocratico. La riprova è offerta dal privilegio per l'ufficio di custode, che il De Rosa non ha nemmeno menzionato tra i suoi incarichi, ma che consente di aggiungere un ruolo completamente inedito per il funzionamento della zecca di Napoli nella prima metà del XV secolo. In effetti non si conosceva nemmeno l'esistenza di questo ufficio e si dubitava degli accepta servitia per eum Maiestati fideliter prestista et impensa dal De Rosa.

\section{Appendice documentaria}

Benevento, 7 febbraio 1443.

Alfonso il Magnanimo conferma a Loise De Rosa l'ufficio di custode dei saggi delle monete d'oro, d'argento e rame coniate nella zecca Napoli, carica già concessa da Ladislao e confermata da Giovanna II, con facoltà di farsi sostituire.

La notizia, riportata in Sambon (1891: 337-338), documentò la coniazione di oro nella zecca dell'Aquila negli anni antecedenti il 1458. Il fatto che Aquila potesse battere oro — prosegue - è confermato pure dal successivo trend aureo riferito dal cronista Francesco d'Angeluccio da Bazzano, che raccontò dei ducati d'oro coniati in questa zecca nel 1475. Secondo Giuliani (2015: 108-109) invece, il Sambon avrebbe equivocato sulle due fonti. 
Alfonso el Magnánimo confirma a Loise De Rosa el cargo de guarda de los ensayos de las monedas de oro, plata y cobre acuñadas en la ceca de Nápoles, cargo ya concedido por Ladislao y confirmado por Giovanna II, con facultad a ser sustituido.

-ACA, Cancillería Real, Registro 2902: Privilegiorum Cancilleriae Neapoli I, anni 1440-1444, f. 159v.

Pro Loyse de Rosa

Alfonsus Dei gratia rex. Universis et singulis presentes licteras inspecturis tam presentibus quam futuris. Licet adieccione plenitudo non egeat nec firmitatem exigat, quod est firmum confirmatur, tandem interdum quod robur obtinet non quod necesitas id expostat, sed ut confirmantis sincera benignitas clareat et res geste ad habundantis cautele robur accedat.

Sane nomine pro parte, Łoysit viri nobilis Loysii de Rosa de Neapoli, fidelis nostri dilecti, fuit Maiestati nostre humiliter supplicatum, ut cum olim clare memorie dominus Rex Ladizlaus. Attendens necessaria firmitate devocionis et fidei dicti Loysii ad grata utilia fructuosa et accepta servitia per eum Maiestati fideliter prestista et impensa eidem Loysio, officium custodis assagii seu probe monete auree, argentee et heree cudende in Sicla civitatis nostre Neapolis, ad vitam suam cum gagijs consuetis et debitis, cum potestate in dicto officio substituendi per idoneum substitutum sufficientem et legalem, de quo sit nominato confidendum graciose concessit prout in ipsis regijs litteris plenius et suisquibus continentiis et deinde clare memorie domina Regina Johanna secunda mater nostra Reverenda. Attendens tunc grata servitia per eum Maiestati fideliter prestita et impensa dictum officium cum gagijs supradictis et potestate substituendi eidem Loysio, prefacta vita durante, confirmavit, prout in quibusdam litteris ditte domine Regine in nostra Curia originaliter productis et presentatis visisque et lectis, sub datis in Castro Novo civitatis Neapolis anno domini $\mathrm{MCCCCXX}^{\circ}$ die $\mathrm{XI}^{\circ}$ mensis februarii XIII ${ }^{\mathrm{e}}$ indictionis hec et alia plenius et seriosus continentur. Demum vero, nos attendentes necessaria et servicia ipsius Loysii nostre Maiestati fideliter prestita et impensa per magnus intendentes gesta per dictam dominam Reginam matrem nostram firmam persistere eidem Loysio dictum officium cum prefatis gagijs, cum lucris et debitis ac cum postestate substituendi prepositum substitutum confirmamus, prout in nostris litteris in nostra Curia productis et presentatis visisque et lectis sub datis in monasterio civitatis Gayete, die VIII $^{\circ}$ mensis augusti anno a Nati- 
vitate Domini $\mathrm{M}^{\circ} \mathrm{CCCC}^{\circ} \mathrm{XXII}^{\circ}$ continetur. Quarum litterarum vigore ipse Loysius ac presentis concessionis iamdicte sive in possessione seu quasi prout et ad presens existit dignaremur sibi dictum officium cum prefatis gagijs, cum potestate substituendi una cum dictis litteris graciosis confirmare. Notandum hiram super premissis cum Consilii deliberacione matura moti nihilominus in hac presente premissis supplicacionibus dicti Loysii nostro culmini prorectis et alias attendentes ipsius Loysii iusta sincera devocione et fide grata quoque grandia utilia fructuosa et accepta servicia per eum Maiestati Sue prestita et impensa ad quem prestanda continue se disponit volentesque propterea ab eo agere graciose eidem Loyse, dicta sua vita durante, dictum officium custodie assagii seu probe monete auree et argentee et heree, cum potestate in dicto officio substituendi per idoneum substitutum, de quo sit nominato confidendum et de cuius excessibus et defectibus Loysius ipse littere Curie principaliter teneat, cum prefatis gagijs consuetis et debitis, iuxta ipsarum harum continentias et tenores propiasque litteras ac omnia et singula contenta in illis quibus litteris presens valida firmitatem persistant ad maioris tamen cautele suffragium quod iustitie prodesse et non efficaciter consuevit eo modo, et prout in possessione sint et ad presens existit tenor presentium, de certa nostra scientia, cum dicta nostra Consilii deliberatione matura, confirmamus, ratificamus, acceptamus et aprobamus nostreque potestatis et auctoritatis presidio conmittimus. Mandantes propterea earundem tenore presentium, de certa nostra scientia, magistris et gabellotis seu credencierijs dicti Regni nostri Sicilie eorumque locatenentibus presentibus et futuris, quatenus forma prefatarum et presentium nostrarum confirmatarum litterarum diligenter attenta illam ipsi et eorum quatenus suorum officiorum temporibus eidem Loysio, dicta sua vita durante, consuevit inviolabiliter et faciat ab alijs prout ad nos spectat et spectabit tenaciter et effectualiter observari, quibuscunque prefata gagia consueta et debita proibeant atque solvari ac exiberi et solvi mandent et faciant integre et sine diminucione quacunque, et contrarium non faciant sicut nostram gratiam caram habent indignacionemque cupiunt evitare. Quibuscunque ordinationibus, litteris, privilegijs, edittis, comissionibus, cedulis et mandatis et alijs presentibus sorte gratijs, quibus omnibus in quantum presentibus refragant totaliter, derogamus et derogatum esse volimus terminus, et iubemus ac etiam quibuscunque concessionibus quibusuis heredibus et personis exinde concessis et factis sub quacunque forma et expressione litterarum, non obstantibus quoquomodo in cujus rei testimonium presentes litteras exinde fieri et magno Maiestatis nostre pendenti sigillo iussimus muniri. Datum in civitate nostra 
beneventana, die $\mathrm{VII}^{\circ}$ mensis februarii, VIa indictione. Anno Domini $\mathrm{M}^{\circ}$ CCCC XXXXIII ${ }^{\circ}$ Regnorum nostrorum anno vicesimo ottavo hujusmodi Regni nostri Sicilie citra Farum Anno Nono ${ }^{\circ}$ Rex Alfonsus.

Dominus Rex mandavit mihi Arnaldo Fonolleda et vidit Ban[c] Baptista Plathamei

\section{Fonti archivistiche}

AGV, Títulos y Enajenaciones, vol. II, anno 1417, ff. 228-230.

Ordenacio de les coses que son necessaries en tota seca i de ffondicio per fer la moneda menuda o d'argent.

ACA, Cancillería, Alfonso el Magnánimo (Benevento, 7 febbraio 1443); f. 159v.

Pro Loyse de Rosa

Compte del Banc d.en Mirabal

AGV, leg. 436, Ap. Varia 1955, n. 208. 17 aprile 1447.

Comptes de G. Miroballo o Llibre de comptes de Mateu Pujades, tresorer reial, i Giovanni Miraballo, banquer de Nàpols.

LLIBRE DE COMPTES DE LA SECA DE NÀPOLS

ACA, Real Patrimonio de Cataluña, Maestre Racional, Volúmenes, Serie General, 2011, ff. 1r-60r. Napoli, 22/2/1453-31/8/1454.

Libro de cuentas de la seca de Nápoles, de Francisco Singniere, maestre de la seca.

ASNA, RCS, Executoriarum, anni 1442-1460.

ASNA, RCS, Significatoriarum, I (Napoli, 3 agosto 1467), ff. 195v-196r.

AGS, Visitas de Italia, leg. 16, exp. 17, Instrucciónes para la cecca de la moneda ann 1543, 1546 y 1561.

AGS, Visitas de Italia, leg. 78, exp. 22, año 1584

\section{Bibliografia}

Bentley, Jerry H. (1995), Politica e cultura nella Napoli rinascimentale. Napoli, Guida Editori.

Bovi, Giovanni (1968), «Le monete di Napoli dal 1442 al 1516», Bollettino del Circolo Numismatico Napoletano, 53: 3-65.

CROCE, Benedetto (1913), «Sentendo parlare un vecchio napoletano del Quattrocento», Archivio Storico per le Province Napoletane, 28: 260-277.

CRUSAFONT I SABATER, Miquel (2015), Història de la moneda catalano-aragonesa medieval (Excepte els comtats catalans), (1067/1162-1516). Barcelona, Societat Catalana d'Estudis Numismàtics. 
D’AdDosio, Giovan Battista (1889), Sommario delle pergamene conservate nell'archivio della Real Santa Casa dell'Annunziata di Napoli. Napoli, Barnaba Cons. di Antonio.

DE BLASIIS, Giuseppe (1879), «Tre scritture napoletane del secolo XV», Archivio Storico per le Province Napoletane, 4, fasc. 3: 411-467.

De Nichilo, Mauro (1991), «Loise de Rosa», Dizionario Biografico degli Italiani, 39. Ed. on-line.

FORMENTIN, Vittorio (1998), Ricordi: edizione critica del ms. Ital. 913 della Bibliotheque de France di Loise de Rosa. 2 voll. Roma/Salerno, Salerno Editrice.

GIULIANI, Achille (2015), «Il privilegio di zecca del conte di Montorio Luigi Camponeschi. Un singolare caso di feudalità monetaria nell'Aquila aragonese», Bollettino del Circolo Numismatico Partenopeo, 2: 95-127.

IADANZA, Giuseppe (1952), «Un fiorentino alla corte di Giovanna II d'Angiò-Durazzo: Gaspare Bonciani», Archivio Storico per le Province Napoletane, 32: 1-20.

LAWSON, William John (1857), «Storia de’ banchi nella Gran Bretagna», Moneta e suoi surrogati, 6: 663-911.

LÓPEZ RodríGuEZ, Carlos; PALMIERI, Stefano (2018), I registri Privilegiorum di Alfonso il Magnanimo della serie Neapolis dell'Archivio della Corona d'Aragona. Napoli, Nella Sede dell'Accademia Pontaniana.

MATEU Y LLOPIS, Felipe (1937), «El vocabulari medieval de l'exercici de la monederia segons documents valencians», Butlletí de dialectologia catalana, 24: 98-129.

MosCATI, Ruggero (1953), «Ricerche su gli atti superstiti della cancelleria di Alfonso d'Aragona», Rivista Storica Italiana, 65: 540-552.

NAVARRO ESPINACH, Germán; IGUAL LUIS, David (2002), La tesoreria general y los banqueros de Alfonso Vel Magnànimo. Castellón de la Plana, Sociedad Castellonense de Cultura.

PALMIERI, Stefano (2018), «Introduzione.» En Carlos López Rodríguez e Stefano Palmieri, I registri Privilegiorum di Alfonso il Magnanimo della serie Neapolis dell'Archivio della Corona d'Aragona. Napoli, Nella Sede dell'Accademia Pontaniana: $\mathrm{V}-\mathrm{X}$.

Perfetto, Simonluca (2012), La prova del metallo: l'esperienza di Vincenzo Porzio nella zecca di Napoli (1555-1587). Roma, Aracne.

Perfetto, Simonluca (2015), La unitat monetària de les Dues Sicílies pel català Francesc Ximenis. La magistratura de la seca i el Llibre de Comptes de la seca de Nàpols (1453-1454). Ariccia (RM), Ermes. Amb la col·laboració de la Societat Catalana d'Estudis Numismàtics (Institut d'Estudis Catalans).

Perfetto, Simonluca (2016), «L'altro volto di Benedetto Cotrugli, il mercante umanista», Italica Belgradensia, 1: 9-17.

Perfetto, Simonluca (2016a), «Salvatore de Ponte, uno dei mastri di zecca che durante il regno di Ferrante batte sesquiducati a nome del Magnanimo, e la zecca aragonese di Fondi (1460-1461)», Acta Numismàtica, 46: 145-157. 
PERFETTO, Simonluca (2017), «Instrucciónes para la cecca dela moneda de Nápoles ann 1543, 1546 y 1561. Edizione commentata», Quaderno di studi, 12: 107-162.

PERFETTO, Simonluca (2019), «Avemo libre d'ariento il quale metemo in zecha»: I «charlini» postumi battuti a Napoli al tempo di Giovanna II d'Angiò (1414-1435), Rivista italiana di numismatica e scienze affini, 120: 227-268.

PERFETTO, Simonluca (2019a), «Era grandissima confusione che non se posseva ritrovarse quella scriptura che si desiderava e cercava»: il riordino dell'Archivio della Regia Zecca (1545-1562), Archivio Storico per le Province Napoletane, 137: 243280.

Perfetto, Simonluca (2020), La zecca di Napoli al tempo di Federico II, Monete Antiche, 113: 27-46.

Perfetto, Simonluca (2020a), Cronologia analitica degli ufficiali della zecca di Napoli (1503-1546), Quaderno di studi, 15: 7-40.

Petrocchi, Giorgio (1957), Masuccio Salernitano, Il novellino. Con appendice di prosatori del '400. Firenze, Sansoni.

SAMBON, Jules Arthur 1891, «I «cavalli» di Ferdinando I d’Aragona re di Napoli», Rivista Italiana di numismatica e scienze affini, 4: 325-356.

SILVESTRI, Alfonso (1959), «La zecca di Napoli all'inizio della dominazione aragonese». En Studi in onore di Riccardo Filangieri, I: 603-610.

TORRES LÁZARO, Julio (1998), Ordenanzas medievales sobre fabricación de moneda en Castilla. Edición y análisis del vocabulario técnico. 2 voll. Madrid, Universidad Complutense de Madrid.

TravaINI, Lucia 2007, Monete e storia nell'Italia medievale. Roma, Istituto Poligrafico e Zecca dello Stato.

VALL-LLOSERA I TARRÉS, Jordi (2016), La moneda del regne de Nàpols sota sobirania de la Corona catalanoaragonesa 1421-1423/1436-1516 i de la nova dinastia troncal napolitana 1458-1501. Girona. 
